\title{
Jürgen Weitzel
}

\section{Die Konstituierung der Gemeinde aus der Rechtstheorie}

\section{Einleitung}

Zunächst zwei Vorbemerkungen zum Verständnis des Themas:

1. „Konstituierung der Gemeinde“ meint nicht deren Begründung oder Herleitung ausschließlich aus einer wie auch immer gearteten Rechtstheorie, sondern alle Elemente solcher Konzeptionen, die Gemeinden in ihrer Entstehung, Entwicklung und Behauptung fördern und unterstützen.

2. „Rechtstheorie“ meint jede konzeptionelle Rechtsvorstellung, sei sie nun ausdrücklich formuliert oder aber aus dauerhaften Organisationsmustern und einem strukturierbaren rechtlichen Verhalten herleitbar.

Damit zur eigentlichen Einleitung: Sehen wir uns nach Konzeptionen des Rechts um, die für das Gesamtthema der Tagung relevant sein könnten, so kommt einem zunächst die Unterscheidung zwischen gelehrtem und ungelehrtem Recht in den Sinn. Gelehrt wäre dann die Tradition des römisch-kanonischen Rechts, das jedenfalls seit dem 12. Jahrhundert an Universitäten gelehrt wird. Ungelehrt wäre die Vorstellungswelt der nördlich der Alpen angesiedelten Rechtshonoratioren. Nun greift allerdings die Unterscheidung zwischen gelehrtem und ungelehrtem Recht, insbesondere deshalb, weil sie nichts zur Entstehung der Normen aussagt, nicht unmittelbar auf unsere Fragestellung zu. Bei ihr geht es offensichtlich um die Ausbildung und Behauptung der Kommune in einer adligen Umwelt, also um Selbstbestimmung im Gegensatz zu Fremdbestimmung. Rechtskonzeptionen und Rechtselemente, die die Autonomie der Gemeinde stärken, sind also gegen solche abzusetzen, die in Bezug auf die Gemeinde heteronom sind, Fremdeinflüsse auf sie ermöglichen. Der Gesichtspunkt autonomen oder heteronomen Rechts findet eine angemessene Berücksichtigung in der von Wilhelm Ebel ${ }^{1}$ begründeten und vorwiegend vom germanistischen Zweig der Rechtsgeschichtswissenschaft zur Sondierung des Rechtsbegriffs eingesetzten Normentrias von Recht $=$ Rechtsgewohnheit, Einung $=$ Willkür und Gebot $=$ Gesetz. Hasso Hofmann hat

1 Wilhelm Ebel, Geschichte der Gesetzgebung in Deutschland (Göttingen ${ }^{2} 1958$ ); ders., Die Willkür (Göttingen 1953); ders., Der Bürgereid als Geltungsgrund und Gestaltungsprinzip des deutschen mittelalterlichen Stadtrechts (Göttingen 1958). 
diese Typen jüngst sogar als universalgeschichtliche Grundformen des Rechts in Anspruch genommen ${ }^{2}$. In ihnen stehen nicht die Normvermittlung und die Anwendungstechniken des Rechts im Vordergrund der Begriffsbildung, sondern die Elemente, auf die es für die Autonomie der Gemeinde entscheidend ankommt: Normentstehung und Normdurchsetzung. Es sollen also nachfolgend die Rechtsgewohnheit, das Willkürrecht und das Gebotsrecht unter den Gesichtspunkten von Normentstehung und Normdurchsetzung auf ihre Fähigkeit, kommunale Autonomie hervorzubringen, befragt werden. Formulieren wir das Problem im Hinblick auf das Spannungsfeld zwischen Herrschaft und Genossenschaft, so ist leicht zu erkennen, daß sich bei den Rechtsgewohnheiten Herrschaft und Genossenschaft in deren Entstehung und Durchsetzung teilen, daß die Einung Normentstehung und Normdurchsetzung in die Hand der Gemeinde gibt, während das Gebotsrechts nach Entstehung und Durchsetzung einer Herrschaft zuzurechnen ist. Mit Schlußfolgerungen allein aus den als Idealtypen zu verstehenden Rechtskonzeptionen ist jedoch nicht allzuviel gewonnen. Ebenso wie Herrschaft und Genossenschaft gehen sie in der Realität vielgestaltige Verbindungen miteinander ein, die es zu erfassen und im Hinblick auf das Autonomieproblem zu gewichten gilt. Es ist aber auch das Verständnis der drei Normtypen nicht ohne Schwierigkeiten, weshalb diese nachfolgend in aller gebotenen Kürze unter Beachtung der neuesten Entwicklungen - vornehmlich den Historikern - vorgestellt werden sollen.

Ein Teil der Verständnis- und Verständigungsschwierigkeiten ergibt sich daraus, daß die Normentrias von Wilhelm Ebel im Hinblick auf die Verhältnisse nördlich der Alpen konzipiert wurde. Dieser Punkt trifft vornehmlich das mittelalterliche „Recht", also die sowohl südlich wie nördlich der Alpen auftretende ungeschriebene, weder auf einer ausdrücklichen Vereinbarung noch auf einem ausdrücklichen Gebot beruhende Normenmasse. Das in den Quellen nördlich der Alpen als Herkommen, Brauch, Gewohnheit, contume und dergleichen mehr bezeichnete Recht wurde bislang durchweg als "Gewohnheitsrecht" erfaßt und damit den von Schriftlichkeit und zum System tendierender "Rechtsordnung“ als Normalität ausgehenden gelehrten Theorien der consuetudo eingeordnet. Inzwischen dürfte sich die Einsicht durchgesetzt haben, daß das Wesen dieses schriftlosen Rechtes damit verkannt wird. Es wird deshalb nachfolgend auf dem Diskussionsstand des Rechtshistorikertages von 1990, der in dem Sammelband „Gewohnheitsrecht und Rechtsgewohnheiten im Mittelalter"3 seinen Niederschlag gefunden hat, hinsichtlich des mittel-, west- und nordeuropäischen schriftlosen Rechts als von der Rechtsgewohnheit oder den Rechtsgewohnheiten gesprochen. Die Einordnung des in römisch-kanonischer Tradition stehenden wahren Gewohn-

2 Hasso Hofmann, Gebot, Vertrag, Sitte. Die Urformen der Begründung von Rechtsverbindlichkeit (Baden-Baden 1993).

3 Gerhard Dilcher u. a., Gewohnheitsrecht und Rechtsgewohnheiten im Mittelalter (Berlin 1992); vgl. auch die Besprechung von Karl Kroeschell, in: ZRG KA 107 (1990) 335-342 zu Hubert Mordek (Hrsg.), Überlieferung und Geltung normativer Texte des frühen und hohen Mittelalters (Sigmaringen 1986). 
heitsrechts ist nach der jeweiligen Theoriebildung in diesen Bereichen vorzunehmen. Soweit danach Gewohnheitsrecht eine mit dem Schriftrecht gemeinsame Geltungs- und Durchsetzungsstruktur aufweist oder gar der - wenn auch nur fiktiven - Aufnahme in den Willen eines Gesetzgebers bedurfte, ist es dem Typus des Gebotsrechts zuzuordnen.

Innerhalb der Kategorie des Gebotsrechtes gibt es ferner Schwierigkeiten beim Verständnis der Policey und ihrem Verhältnis zum Recht. Die Policeygebote, -normen und -ordnungen des späten Mittelalters und der frühen Neuzeit werfen in ihrem Ve:hältnis zum zeitgenössischen Recht ganz entsprechende Fragestellungen und Probleme auf, wie sie für das Verhältnis des fränkischen Königsrechts, der Dekrete, Edikte und Kapitularien zu den Volksrechten diskutiert werden ${ }^{4}$. In beiden Epochen wurde ein autoritatives, teilweise durch Konsenselemente abgesichertes Ordnungsinstrumentarium entwickelt, um rechtlich begründete Einzelmaßnahmen durchzusetzen, um aber auch auf das Recht und seine Verwaltung ordnend, bessernd, erneuernd und letztlich fortbildend Einfluß zu nehmen. Im normativen Bereich lag der Schwerpunkt im frühen Mittelalter beim Straf- und Prozeßrecht, in der frühen Neuzeit im Wirtschafts- und Arbeitsleben sowie in der ständischen Sozialordnung. Durch massive Anwendung dieses Instrumentariums entstand jeweils eine komplizierte Gemengelage von Gebotsrecht und Rechtsgewohnheit bzw. einem auf gemeinem Schriftrecht gegründeten Richterrecht. In der Neuzeit wurde das Gebotsrecht zum die Zukunft bestimmenden Gesetz, das heißt der Typus des durch Befehl hervorgebrachten Rechts hat schrittweise die alte Rechtsgewohnheit verdrängt und im 18. Jahrhundert die Strukturen des alten Rechts endgültig überwunden. Damit ist zu derzeit nicht näher bestimmbaren Zeitpunkten schon den Zeitgenossen die Fähigkeit abhanden gekommen, Policey und Recht zu unterscheiden. Erst im 19. Jahrhundert wurde Herrschaft dann wieder geteilt, wurden Gebot und das zum Gesetz gewordene Recht in Deutschland wieder gebunden - diesmal durch innere Mechanismen eines monistischen Staatsund Rechtswesens. In der fränkischen Epoche hingegen hatten sich typologisch gesehen die Rechtsgewohnheiten behauptet.

Es waren bekanntlich die landesherrlichen Policeygebote und -ordnungen, die den Bewegungsspielraum der Gemeinden in der frühen Neuzeit zunehmend einengten. Wie aber ist das gemeine, gelehrte Recht dieser Zeit typologisch einzuordnen? $\mathrm{Da}$ auf dem Corpus Juris Justinians beruhend, ist es grundsätzlich als Gebots- und Gesetzesrecht zu bewerten. Allerdings handelt es sich um ein Gesetzesrecht besonderer Art. Es ist nämlich das Recht eines längst verblichenen Gesetzgebers, der nicht mehr befragt werden kann, der sein Werk vielmehr in die Hand der Wissenschaft und in die Durchsetzungspraxis der Richter entlassen hat. Das heißt, daß seit dem späten Mittelalter neben den Policeygeboten und -gesetzen

\footnotetext{
${ }^{4}$ Jürgen Weitzel, Merkantilismus und zeitgenössische Rechtswissenschaft, in: Städtewesen und Merkantilismus in Mitteleuropa, hrsg. von Volker Press (Köln 1983) 45-81, 58 ff.; ders., Strafe und Strafverfahren in der Merowingerzeit, in: ZRG Germ. Abt. 111 (1994) 66-147, $76 \mathrm{ff}$.
} 
über Jahrhunderte hin ein ausgesprochen schwach akzentuiertes Gebotsrecht den Übergang zum modernen Gesetzesrecht vorbereitete.

Die Einleitung abschließend, soll noch darauf hingewiesen werden, daß nicht nur die Rechtstheorien für Ausbildung und Bestand der Gemeinden von Bedeutung gewesen sind, sondern daß auch umgekehrt vornehmlich die Erfordernisse des städtischen Sozial- und Wirtschaftslebens auf die Konzeptionen des zeitgenössischen Rechts eingewirkt haben. Wilhelm Ebel $^{5}$ und zuletzt Gerhard Dilcher ${ }^{6}$ haben nachdrücklich auf die Vorbereitung der Rezeption des gelehrten Rechts durch das deutsche städtische Statutarrecht hingewiesen. Mit der in der Stadt früher und nachhaltiger als auf dem Land einsetzenden Verschriftlichung, mit polizeilichen Elementen im Statutarrecht und mit zunehmender autoritativer Aufladung des Statutarrechtes selbst haben die Kommunen ihrerseits die Entwicklung hin zum Gebotsrecht mitgetragen.

\section{Rechtsgewohnheit und Kommunen}

Rechtsgewohnheiten entstehen in der Regel aus konsentierter Übung. Die Übung als solche ist jedoch nicht Geltungsgrund des Rechts, sondern nur seine Erkenntnisform. Das Recht gründet im gegenwärtigen Konsens der ständischen Gruppe, um deren Angelegenheiten es geht, also der Rechtsgenossen. Für diese Grundlagen beziehe ich mich auf meine 1985 erschienene Schrift „Dinggenossenschaft und Recht" ${ }^{\text {"7 }}$ sowie auf weitere Beiträge bis hin zu dem Vortrag über „Gewohnheitsrecht und fränkisch-deutsches Gerichtsverfahren", der 1992 in dem vorgenannten Sammelband über "Gewohnheitsrecht und Rechtsgewohnheiten im Mittelalter" erschienen ist ${ }^{8}$. Danach ist die Trennung von Urteil (= Recht, Rechtsfindung) und Gebot (= Rechtszwang) Ausdruck eines qualitativen Unterschiedes zwischen mittelalterlich-deutschem Recht und neuzeitlich-autoritativem Gesetzesrecht. In der gerichtlichen Organisation und im Verfahrensrecht findet die Struktur der Rechtsgewohnheit ihren Ausdruck, sobald das Recht streitig geworden ist. Die Dinggenossenschaft in Gericht und Rechtsdurchsetzung meint den Zustand der Trennung von Rechtsfindung (= Urteil = Entscheidung) und richterlichem Rechtszwang (= Gebot des Rechts) unter maßgeblicher Beteiligung der Genossen der Parteien an der Rechtsfindung. Im mittelalterlichen deutschen Gericht ver-

5 Wilhelm Ebel, Die rechtschöpferische Leistung des mittelalterlichen deutschen Bürgertums, in: Die gesellschaftliche Struktur der mittelalterlichen Städte in Europa (VuF XI, Sigmaringen 1966) 241-258.

6 Gerhard Dilcher, „Hell, verständig, für die Gegenwart sorgend, die Zukunft bedenkend“. Zur Stellung und Rolle der mittelalterlichen deutschen Stadtrechte in einer europäischen Rechtsgeschichte, in: ZRG GA 106 (1989) 12-45.

7 Jürgen Weitzel, Dinggenossenschaft und Recht. Untersuchungen zum Rechtsverständnis im fränkisch-deutschen Mittelalter (Köln 1985).

${ }^{8}$ Dilcher, Gewohnheitsrecht (wie Anm. 3), 67-86. 
waltet also die Gerichtsgemeinde, der Verband der Rechtsgenossen, sein Recht inhaltlich selbst. Die Selbstbestimmung formt sich dabei nur in dem als selbstverständlich akzeptierten Ordo der mittelalterlichen Stände aus. Dieses Regelungsmodell überfordernde Konflikte führen zu Gewalt, u.U. zu neuen Formen der Vergesellschaftung. Die genossenschaftliche Struktur der Rechtsbildung und der Streiterledigung ist meines Erachtens ein glückliches Produkt aus der frühmittelalterlichen Verschmelzung von germanischen und römischen Grundvorstellungen. Sie hängt wesentlich damit zusammen, daß die Franken und andere germanische Stämme genossenschaftliche Formen der Willensbildung und Entscheidungsfindung (Sühneverhandlungen und Sühnevertrag) kannten, und daß mittelalterliche Herrschaft niemals so stark war, daß sie konkurrierende Herrschaftsträger hätte ausschließen oder nachgeordnete Verbände über die durch den Ordo vorgegebenen Grundzüge hinausgehend hätte reglementieren können. Andere haben den genossenschaftlichen Grundzug der Rechtsgewohnheit aus dem Lehnswesen? oder gar aus dem ethnologischen Typus der „Gabe ${ }^{10}$ herleiten wollen. Beides überzeugt mich nicht.

Ist nun die dinggenossenschaftlich organisierte Gerichtsgemeinde des mittelalterlichen Rechts der "Mutterboden“ der Kommunen? Man kann und muß dies eindeutig verneinen. Das Gericht integriert Herrschaft und Genossenschaft. Es ist nicht darauf angelegt, sämtliche Belange der Rechtsgenossenschaft in Eigenregie zu betreiben. Selbstbestimmt ist nur die Aussage über den Inhalt des Rechts als solche. Bereits die Einberufung der Gerichtsversammlung geht grundsätzlich vom Herrschaftsträger aus. Er übernimmt den Vorsitz in der Versammlung, leitet sie, gebietet das von den Rechtsgenossen gefundene Urteil und sorgt gegebenenfalls mit Zwang für dessen Durchsetzung. Nur als Systembruch zugunsten der Kommune wurden Bestrebungen unternommen, den vorsitzenden Herrschaftsträger abzuschaffen oder ihn durch ein Mitglied der Gerichtsgemeinde zu ersetzen. Die Gerichtsgemeinde hat also nicht von sich aus zur "Gegnerfreiheit" tendiert, suchte nicht eine Form autonomer politischer Organisation im Gegensatz zum Adel zu werden. Ein weiterer grundlegender Einwand geht dahin, daß sich die Gerichtsgemeinde trotz der bei der Rechtsfindung unterlaufenden Rechtsfortbildung nicht als ein Organ zur Schaffung neuen Rechts, sondern als ein solches der Darstellung und Bewährung von Recht verstand. Herleitungen der Entwicklung des Gemeindewesens aus der mittelalterlichen Gerichtsverfassung, wie sie z. B. Franz Steinbach ${ }^{11}$ für die niederrheinischen Kommunen vertreten hat, ist daher grundsätzlich mit Skepsis zu begegnen. Freilich kann unter besonderen Bedingungen das Vorbild der Gerichtsgemeinde einen über dem Üblichen liegenden Einfluß genommen haben, denn mit der Aussage, daß die Dinggenossen-

9 Walter Ullmann, Individuum und Gemeinschaft im Mittelalter (Göttingen 1974) 40-73.

10 Heide Wunder, Die bäuerliche Gemeinde in Deutschland (Göttingen 1986). Mit Relevanz zum Typus der „Gabe“ vgl. aus rechtshistorischer Sicht zuletzt Franz Dorn, Die Landschenkungen der Merowinger (Paderborn 1991).

11 Franz Steinbach, Stadtgemeinde und Landgemeinde, in: Rh. VjBll. 13 (1948) 11-50; ders., Der Ursprung der Kölner Stadtgemeinde, in: Rh. VjBll. 19 (1954) $273 \mathrm{ff}$. 
schaft nicht der „Mutterboden“ der Kommunen gewesen sei, soll ja dem Vorbild der Gerichtsversammlung nicht jegliche Bedeutung für unsere Fragestellung abgesprochen sein. Bevor ich auf den mittelbaren Rückhalt der Kommunen im Recht zu sprechen komme, möchte ich jedoch noch zwei weitere Gesichtspunkte thematisieren, die die negative Grundeinschätzung tragen.

Zum ersten geht es um die geographische Verbreitung der Dinggenossenschaft und damit eines Rechtsverständnisses, das zwischen genossenschaftlicher Rechtsbildung und Streiterledigung einerseits und herrschaftlicher Durchsetzung des Rechts andererseits unterscheidet. Diese Struktur der Rechtsgewohnheit ist neben der bereits erwähnten Herrschaftsschwäche wesentlich der Mündlichkeit dieses Rechtstypus geschuldet. Für das Mittelalter ist also, neben anderen Unterscheidungen, in Europa von zwei großräumigen unterschiedlichen Rechtskonzeptionen auszugehen: dem Gebiet einer grundsätzlich schriftlichen und in Kategorien der Schrifterfahrung denkenden Rechtskultur und dem Gebiet einer grundsätzlich in der Mündlichkeit ruhenden Rechtskultur. Dem Vorherrschen des Schriftrechtes entspricht die Einheit - zumindest die Einheit des konzeptionellen Verständnisses - von Rechtsbildung, Rechtsfindung und Rechtszwang sowie die latent vorhandene Nähe und Bereitschaft zum Gesetzesrecht. Die prinzipiell mündliche Rechtskultur findet ihren Ausdruck in der Dinggenossenschaft sowie in ihren über Jahrhunderte hin und bis weit in die frühe Neuzeit hinein bestehenden Schwierigkeiten, den Übergang zum autoritativen Gesetzesrecht zu finden. Im Bereich des Schriftrechtes haben wir es mit Nachwirkungen der antiken Rechtskultur zu tun ${ }^{12}$, wie sie in ähnlicher Weise außerhalb geographischer Festlegungen weite Teile des Kirchenrechts charakterisierten. Für das weltliche Recht ist die Unterscheidung zwischen dem südfranzösischen Schriftrecht und den nordfranzösischen coutumes allgemein bekannt. Die etwa von der Ile d'Oléron nach Genf laufende Linie ist jedoch über Trient bis hin nach Istrien zu verlängern, so daß danach Europa in einen südlichen Bereich des grundsätzlich autoritativen Schriftrechts und einen nördlichen Bereich der grundsätzlich genossenschaftlichen Rechtsgewohnheit geschieden ist ${ }^{13}$. Da nun die italienischen Kommunen stark und frühzeitig entwickelt sind, Italien aber in der Sache nicht dinggenossenschaftlich geprägt war ${ }^{14}$, erscheint es ausgeschlossen, das Kommunewesen aus dem Typus der dinggenossenschaftlichen Rechtsgewohnheit herzuleiten. Die italienische Rechtsfindung bediente sich, von punktuellen Ausnahmen, insbesondere solchen des Lehnrechts, abgesehen, immer sachkundiger Urteiler (meist iudices genannt) und nicht des Urteils von Vertretern der Gerichtsgemeinde oder gar des Urteils der Gerichtsgemeinde selbst.

Zum zweiten ist auf die Trennung von überkommenem Gericht und kommuna-

$12 \mathrm{Vgl}$. auch Gerhard Köbler, Vorstufen der Rechtswissenschaft im mittelalterlichen Deutschland, in: ZRG GA 100 (1983) 75-118; Charles M. Radding, The Origins of Medieval Jurisprudence. Pavia and Bologna 850-1150 (New Haven 1988).

${ }_{13} \mathrm{John}$ Gilissen, Introduction, historique au droit (Brüssel 1979) 165, 241; Weitzel, Dinggenossenschaft (wie Anm. 7), 338, 478.

14 Weitzel, (wie Anm. 7), 151f., $331 \mathrm{ff}$. 
lem Willkürorgan in den Kommunen hinzuweisen ${ }^{15}$. Besonders ausgeprägt ist dieser Unterschied in den Städten und dort wiederum seit der Durchsetzung der Ratsverfassung, die in Deutschland im wesentlichen in das 13. Jahrhundert fällt. Das überkommene stadt- bzw. landesherrliche Gericht, das in dinggenossenschaftlichen Formen urteilte, bestand in den deutschen Stadt- und Landgemeinden im Prinzip während des gesamten hohen und späten Mittelalters neben der kommunalen Gerichtsbarkeit fort. Das Verhältnis war immer das einer Spannung und Konkurrenz zwischen zwei Rechtsprechungskörpern bzw. Kompetenzbereichen. Grundsätzlich trachteten die Städte dahin, die stadtherrliche Gerichtsbarkeit zu erwerben, um sie dem Bereich der autonomen Stadtregierung einzugliedern. Die Formen, in denen dies geschah, sind zu vielgestaltig, als daß sie hier vorgestellt werden könnten. Wo die Übernahme gelang, was keineswegs überall der Fall gewesen ist, blieb doch die Form der dinggenossenschaftlichen Rechtsfindung als solche erhalten. Es ist deshalb keineswegs erstaunlich, daß selbst ausgangs des Mittelalters in großen Städten, die die alten Gerichte usurpiert hatten, ein Bewußtsein dafür bestand, daß der Rat nicht eigentlich, d.h. im Sinne des (Land-)Rechts, Träger von Gerichtsbarkeit sei. Erst die rezipierte Gerichtsverfassung der frühen Neuzeit hat diese Unterschiede eingeschmolzen. Auch diese Zusammenhänge sind ein eindeutiger Hinweis darauf, daß sich die Kommune zumindest partiell in einem ausgesprochenen Widerspruch zu Rechtsgewohnheit und Dinggenossenschaft entwickelt und behauptet hat.

Andererseits steht jedoch außer Frage, daß die genossenschaftliche Struktur der Rechtsgewohnheit die weiter ausgreifende kommunale Genossenschaft in vielfältiger Weise mittelbar gestützt und auch ihrer Ausbildung vorgearbeitet hat. Zunächst ist die Existenz einer Rechts- und Gerichtsgemeinde als solche ein Faktor, der das Selbstbewußtsein der Rechtsgenossen stärken, ihre Bereitschaft, sich zur Wahrnehmung auch anderer Angelegenheiten als der Rechtsfindung zu versammeln, fördern mußte, so weit nur der herrschaftliche Verband, dem sie angehörten, dazu überhaupt Spielräume eröffnete. Eine Bevölkerung, die es gewohnt war, in Versammlungen zur Wahrnehmung ihres Rechts zusammenzutreten, konnte tendenziell auch mehr, als nur die Rechtsfindung in die eigene Hand nehmen. Gerichtsversammlung, insbesondere das ungebotene, echte Ding, und Gemeindeversammlung mögen denn auch nicht selten in einem Zuge abgehalten worden sein. Darüber hinaus war die Ausbildung von dinggenossenschaftlich organisierten Hofgerichten in früh- und hochmittelalterlichen Grundherrschaften nicht nur Ausdruck einer verbesserten rechtlichen Stellung des einzelnen abhängigen Bauern (Überwindung des im Prinzip antiken Sklavenstatus), sondern führte zugleich die Voraussetzungen dafür herauf, daß späterhin unter Aushöh-

15 Wilfried Helling, Untersuchungen zur Entwicklung der mittelalterlichen bremischen Stadt- und Gerichtsverfassung (Diss. iur. Kiel 1959); Wilhelm Ebel, Lübisches Recht (Lübeck 1971) 318-381; Weitzel, (wie Anm. 7), 1146, 1314-1326; Pirmin Spieß, Willkür, Statuten und Landesherrschaft in der spätmittelalterlichen Stadt Südwestdeutschlands, in: Statuten, Städte und Territorien zwischen Mittelalter und Neuzeit in Italien und Deutschland, hrsg. von Giorgio Chittolini und Dietmar Willoweit (Berlin 1992) 325-342. 
lung älterer Hofverbände Dörfer und ländliche Siedlungen zu Gemeindeverbänden werden konnten.

In diesem Zusammenhang ist insbesondere für den Bereich der Stadt darauf hinzuweisen, daß als Führungsgruppe der entstehenden Kommunen an Rhein, Maas und Mosel häufig das Schöffenkollegium genannt wird ${ }^{16}$. Es ist dies jedoch auch in Magdeburg bis 1244, also vor der Entstehung der Ratsverfassung, der $\mathrm{Fall}^{17}$. Über die gesellschaftliche Kennzeichnung der Führungsgruppe als Kaufleute und allmählich verbürgerlichende Ministeriale hinaus kann also mit dem Schöffenkollegium ein rechtlich-organisatorisches Element in der Frühgeschichte der mitteleuropäischen Stadt dingfest gemacht werden.

In der frühen Neuzeit stützt die Konzeption der Rechtsgewohnheit die Kommunen zunächst noch gegen landesherrliche Ambitionen. Diese sind nun vom rezipierten Herrschafts- und das heißt zunächst einmal Rechts- und Richterverständnis getragen. Genossenschaft überlebt tendenziell dort, wo ihre Regelungsgegenstände für den Landesherren uninteressant sind oder wo genossenschaftliche Willensbildungs- und Entscheidungsformen zugunsten der landesherrlichen Verwaltung eingesetzt werden können. Das überkommene Rechtsverständnis und die genossenschaftliche Rechtsfindung zählen zu den Faktoren, die nur allmählicher Umgestaltung zugänglich waren. Insbesondere die großen Städte hatten diese Faktoren jedoch schon selbst geschwächt. So blieb das alte Recht eher in den Landstädten und Landgemeinden ein retardierendes Element gegen den entstehenden Territorialstaat. Besonders anschaulich wird die gemeinsame Niederlage von Recht und Gemeinde in Brandenburg um 1500. Die sich selbständig gebenden Städte hat der Markgraf schon im 15. Jahrhundert teilweise mit Gewalt wieder in die landesherrliche Gerichtsverfassung eingebunden und damit auch den Einwirkungen der Rezeption ausgesetzt. Im Jahre 1516 wird dann das gemeine römische Recht für allgemein verbindlich erklärt. Damit setzt sich dessen Gerichtsverfassung, das gelehrte Einzelrichtertum, auch in den Landgemeinden gegen die herkömmlichen Schöffengerichte durch. Aus dieser Verdrängung ziehen die Gutsbesitzer ihren Vorteil, indem sie die Patrimonialgerichtsbarkeit etablieren ${ }^{18}$. Insgesamt aber ist im ländlichen und kleinstädtischen Bereich die dinggenossenschaftliche Rechtsfindung im 16. Jahrhundert noch weit verbreitet. Den Umbruch bringt das 17. Jahrhundert. Ausläufer finden sich bei Landgemeinden bis weit in das 18. Jahrhundert hinein ${ }^{19}$.

16 Edith Ennen, Die europäische Stadt des Mittelalters (Göttingen 1972) 135-138.

17 Jürgen Weitzel, Zum Rechtsbegriff der Magdeburger Schöffen, in: Studien zur Geschichte des sächsisch-magdeburgischen Rechts in Deutschland und Polen, hrsg. von Dietmar Willoweit und Winfried Schich (Frankfurt a.M. 1980) 62-93, 77 f.

${ }^{18}$ Hartmut Harnisch, Die Landgemeinde im ostelbischen Gebiet (mit Schwerpunkt Brandenburg), in: Landgemeinde und Stadtgemeinde, HZ Beiheft 13, hrsg. von Peter Blickle (München 1991) 309-332, $324 \mathrm{ff} ., 331$.

19 Werner Troßbach, Die ländliche Gemeinde im mittleren Deutschland, in: Landgemeinde und Stadtgemeinde (wie Anm. 18), 263-288, 273f.; Götz Landwehr, Die althannoverschen Landgerichte (Hildesheim 1964). 


\section{Willkürrecht (Statutarrecht) und Kommunen}

\section{Gemeineuropäische Grundlagen}

Ob man nun nach Norditalien, nach Nordfrankreich und Flandern oder nach Deutschland blickt, allenthalben entsteht oder versteht sich die Stadtgemeinde als ein unter Gleichen geschlossener Schwurverband, dem kraft der eingegangenen eidlichen Verbindung die Befugnis zur Regelung seiner eigenen Angelegenheiten zusteht. Wie unterschiedlich die Wege, die zur Ausbildung von Stadt- und Landgemeinden führten, auch immer waren, so ist doch die Charakterisierung der Kommunen durch die eidliche Verbindung der sie tragenden Personen allgemeine Auffassung ${ }^{20}$. Allein darauf scheint es mir bei der hier verfolgten Fragestellung anzukommen. Die eidliche Verbindung der Bürger, sei es in der ursprünglichen conjuratio, sei es im später geleisteten Bürgereid, trug eine neue „Rechtstheorie“ in sich. Der Bürgereid war, wie es Wilhelm Ebel in seiner bekannten Schrift formuliert hat, Geltungsgrund und Gestaltungsprinzip des deutschen mittelalterlichen Stadtrechts. Und es galt dies offensichtlich nicht nur für die deutschen Kommunen. Man kann die Fragestellung nun nach zwei Seiten hin vertiefen. Zum einen wäre nach dem Wesen des Eides, zu fragen, der in der Lage ist, eine neue normative Ordnung zu begründen. Es handelt sich offensichtlich bei der Fähigkeit des Eides, Gruppen von Menschen zu vergesellschaften, um ein aus der Antike überkommenes, christlich überformtes gemeineuropäisches Phänomen ${ }^{21}$, das über den Einsatz der gesamten Persönlichkeit zur Erreichung der gemeinsamen Ziele hinausgehend, letztlich in transzendentalen Vorstellungen von der Wirkung des Eides gründet. Selbst der Kaiser, die Könige, die Fürsten und sonstige Gegner der städtischen Schwurverbände haben dies nicht bestritten. Sie haben vielmehr die conjurationes verboten und als ein "Geschwür am Volkskörper, einen Schrekken für das Reich, einen Schauder für die Geistlichkeit" bezeichnet ${ }^{22}$. Dieser Aspekt soll hier jedoch nicht weiter vertieft werden.

Zum anderen wäre nach der Entfaltung der im Eid angelegten Gestaltungsmöglichkeiten zu fragen. Denn selbstverständlich hatte nicht jede conjuratio derart weitgehende, aus dem Recht hinausführende Konsequenzen. Eidliche Verbindungen zum Zwecke der Wahrung des Landfriedens oder zur Sicherung der Rechte von Klerikern gegenüber ihren Bischöfen tendierten eben nicht zur typologischen Überwindung des jeweils maßgeblichen Rechts. Erst die Selbstorganisation wirtschaftlich und politisch so außergewöhnlich entwicklungsfähiger Personengruppen ließ die entstehenden Gemeinschaftsregeln allmählich unter Einschmelzung weiterer normativer Elemente zu einem neuen Typus des Rechts, eben des auf

${ }^{20}$ Ennen, (wie Anm. 16); Gerhard Dilcher, Die Entstehung der lombardischen Stadtkommune (Aalen 1967); Knut Schulz, „Denn sie lieben die Freiheit so sehr...". Kommunale Aufstände und Entstehung des europäischen Bürgertums im Hochmittelalter (Darmstadt 1992).

2) Dies haben die Untersuchungen von Otto Gerhard Oexle aus den letzten Jahren erwiesen.

22 Ennen, (wie Anm. 16), 124. 
Willkür beruhenden Stadtrechts werden. Die Dörfer brachten zwar auch Willküren hervor, konnten diese jedoch nicht als eigenen Rechtskreis gegen das Landrecht durchsetzen.

Das Willkür- oder Statutarrecht, ist also das der Kommune „arteigene“ Recht. Es geht aus einer autonomen Verbandsgewalt hervor und korrespondiert der Grundlage der Gemeinde in der conjuratio. Die Betrachtung der Entwicklung dieses neuen Rechts, insbesondere auch die Frage, ob und wie das städtische Recht die in der conjuratio vorgegebenen Grundlagen ausgeformt und auf Dauer eingelöst hat, muß für die Landschaften der Rechtsgewohnheit einerseits, des Gewohnheitsrechts andererseits getrennt untersucht werden.

\section{Willkïrrecht und Kommunen im Bereich der Rechtsgewobnheit (coutumes)}

Was zunächst die Normbildung angeht, so war die Konzeption des Willkürrechts, setzen wir ihre bewußte Erfassung und Instrumentalisierung gegen die Kräfte des Landrechts und gegen die Einflußnahme des Stadtherrn auf die erste Hälfte des 12. Jahrhunderts an, ein revolutionärer Akt. Das Revolutionäre besteht von der Rechtskonzeption her gesehen darin, daß hier erstmals in einer unmittelbar sozialrelevanten Form und einer nicht unbedeutenden Größenordnung der Anspruch formuliert wird, die Sozialordnung durch bewußt gemachte, also gesetzte Regeln zu gestalten. Der städtische Schwurverband war in einer Zeit, die allgemein keine Gesetzgebung, sondern allenfalls die massenhafte Vergabe von Einzelprivilegien kannte $^{23}$, auf generelle Normbildung hin angelegt. Freilich wurde dies von den Herren so nicht zugestanden und von den Bürgern so nicht überall offen propagiert. Gleichwohl zeigt etwa der - verfälschende - Umgang der Lübecker mit den ihnen verliehenen Privilegien um 1200 eindeutig den Willen auf, das Willkürrecht $\mathrm{zu}$ einer einheitlichen Stadtrechtsordnung werden zu lassen ${ }^{24}$.

Das Recht zum Erlaß von Statuten bezog sich zunächst auf die Spezifika der neuen Gemeinwesen, als da sind: Angelegenheiten des Marktes, kaufmännische Gewohnheiten, Maß und Gewicht, Lebensmittelpolizei, Streitigkeiten der Kaufleute und Bürger untereinander, die Wahrung von Ordnung und Frieden auf dem Markt und in der Stadt. Es galt nun, die Kompetenz zur Setzung von Willküren gegen das Recht hin zu erweitern. Da Recht nicht direkt zum Gesetz werden konnte, verschafften sich die Bürger und insbesondere ihr Ratsorgan die Befugnis zur Rechtsbesserung durch Willküren. In der Ausbildung eines einheitlichen Willkürrechts sind die Stadt Lübeck und die Städte des lübischen Rechts wohl am weitesten vorangekommen ${ }^{25}$. Neben der Setzung von Willküren haben freilich auch neugebildete Gewohnheiten und die überkommene Rechtsprechung, vornehmlich soweit sie vom Rat beherrscht wurde oder unter seinen Einfluß geriet, zur Ausbildung eines einheitlichen Stadtrechts beigetragen. Die gänzliche Ein-

23 Hermann Krause, Gesetzgebung, in: HRG I, 1606-1620, 1610 ff. mit weiteren Hinweisen.

24 Jürgen Weitzel, Über Oberhöfe, Recht und Rechtszug (Göttingen 1981) 43-50.

${ }_{25}$ Ebel, (wie Anm. 15), 7 ff., $168 \mathrm{ff}$.; ders., (wie Anm. 5). 
schmelzung ist jedoch im Mittelalter in den wenigsten Städten erreicht worden. Erst das gelehrte Recht, seine Statutentheorie und die durch die Appellation begründeten Rechtsmittelzüge haben schließlich die Vorstellung einer einheitlich strukturierten Rechtsordnung durchgesetzt.

Es bleibt noch einiges zur Legitimation des Stadtrechtes nach innen hin zu sagen. Aufgrund des Bürgereides war die Gemeinde in der Theorie eine Gemeinschaft von Gleichen. Durch gewisse Rituale, wie die feierliche Wiederholung des Bürgereides in der jährlichen Gemeindeversammlung, wurde die Vorstellung von der bürgerlichen Gleichheit teilweise bis ins 18. Jahrhundert hinein in Szene gesetzt. In der Praxis der meisten Städte entsprach sie alsbald nach deren Gründung nicht mehr der Wirklichkeit. Bekanntlich haben oligarchische Tendenzen zu einem obrigkeitlichen, von "den Geschlechtern“ beherrschten Stadtregiment geführt, das nur gelegentlich, nur durch Aufruhr und oft nur vorübergehend durch die Zünfte aufgebrochen werden konnte. Im Zuge dieser Entwicklungen wurde der Rat zum Gesetzgebungsorgan, das nicht mehr von allen Bürgern gewillkürte, sondern aus eigener Machtvollkommenheit beschlossene Statuten erließ. Der Bürgereid wurde nun so gedeutet, daß er die Unterwerfung unter die jeweiligen Dekrete des Rates einschloß. Verstärkt wurde diese die Theorie der Gemeinde verlassende Entwicklung durch die dem Rat zukommenden polizeilichen Kompetenzen. Sowohl im Bereich des Marktes als auch hinsichtlich der bei städtischen Wohnverhältnissen viel intensiveren Bemühungen zur Friedenssicherung kamen den Städten von Anfang an Kompetenzen zu, die wir seit der Mitte des 15. Jahrhunderts dann als Policey bezeichnet finden. Soweit es sich um das Marktgeschehen handelt, wurden solche Regelungen oft schon frühzeitig dem Marktrecht zugerechnet. Überwiegend jedoch wurden die Regelungen zur Aufrechterhaltung von Frieden, Sicherheit und Ordnung als Willküren und "Ordnungen" erlassen und angesehen ${ }^{26}$.

Aus beiden Bereichen, dem der Oligarchisierung und dem der Policeygewalt nahmen die städtischen Willküren bereits im Spätmittelalter einen kräftigen Schuß von Gebotsrecht auf. Die genossenschaftliche Gemeindetheorie und die ihr entsprechende Konzeption der Rechtsbildung sind damit weitgehend verlassen. Die nördlich der Alpen gelegenen Städte sind dabei, auf deutschrechtlicher Grundlage Gebotsrecht und Gesetzgebung zu entwickeln. Auch insoweit, nicht nur hinsichtlich der Inhalte des Privat- und Prozeßrechts, haben sie der Rezeption vorgearbeitet. Die Entwicklung des Willkürrechts der Landgemeinden und Dörfer ist so wenig aufgearbeitet, daß hierzu eine allgemeine Aussage nicht vorgetragen werden kann. Schon nach dem Sachsenspiegel war dem (Kolonisten-)Dorf das Recht der Kore nach dem Mehrheitsrecht der Bauern zugelegt ${ }^{27}$. Die Minderheit sollte diesen Beschlüssen nicht widersprechen. Da die Dörfer wohl weniger zur Ausbil-

26 Josef Baader (Hrsg.), Die Nürnberger Polizeiordnungen aus dem XIII. bis XV. Jahrhundert (Stuttgart 1881, Ndr. Amsterdam 1966); Armin Wolf (Hrsg.), Die Gesetze der Stadt Frankfurt am Main im Mittelalter (Frankfurt a.M. 1969).

27 Ssp LdR II 55. 
dung eines Regimentes neigten, da der Regelungsbereich ihrer Willküren weniger ausgedehnt war als der der Städte, zumal er ja auch durch das Landrecht in Grenzen gehalten wurde, ist zu vermuten, daß sich das Strukturprinzip der gemeindlichen Gleichheit auf dem Lande länger erhalten hat als in der Stadt.

Was die Normdurchsetzung angeht, so ist als grundlegend eingangs hervorzuheben, daß Verstöße gegen das Recht vor den überkommenen Gerichten, Verstöße gegen die Willkür jedoch vor den kommunalen Willkürorganen verhandelt wurden. Es gab hier also, dem unterschiedlichen Charakter der beiden Normenbereiche entsprechend, eine im Prinzip strikte Kompetenzteilung, die wie bereits erwähnt, allenfalls dadurch überwunden werden konnte, daß dem kommunalen Willkürorgan Gerichtsherrschaft ausdrücklich übertragen wurde oder aber, daß es - was wohl genauso häufig geschah - die Stellung als Gerichtsherr usurpierte. Letzteres erfolgte meist dadurch, daß der Rat sich als Rechtsbelehrungs- und Rechtszugsspruchkörper über dem Stadtgericht etablierte und dann in den großen Städten nicht selten den weiteren Rechtszug nach auswärts, jedenfalls den Zug an landrechtliche Oberhöfe, an landesherrliche und kaiserliche Gerichte verbot ${ }^{28}$.

Um nun zunächst die Ausführungen zu den Landgemeinden und Dörfern fortzuführen, muß festgestellt werden, daß die Unterscheidung zwischen Gerichtsbarkeit (Urteil; als abstrakte, späterhin auch normative, Aussage Weistum) und der Tätigkeit des Willkürorgans prinzipiell auch auf dieser Ebene gilt. Soweit die Dörfer also die Niedergerichtsbarkeit unter dem Vorsitz eines landesherrlichen Beamten oder eines Dorfvorstehers übten, ist damit noch nichts über die Ausübung der Willkür „gerichtsbarkeit“ gesagt. Über das bäuerliche Willkürverfahren ist jedoch noch weniger bekannt als über das stadtrechtliche. Die Literatur vermittelt den Eindruck, daß zwischen der Tätigkeit des Dorfgerichtes und der des dörflichen Willkürorgans entweder in praxi nicht ausgeprägt unterschieden wurde oder daß die Notwendigkeit einer Unterscheidung den Autoren insoweit gar nicht bewußt ist. Es muß zudem auch davon ausgegangen werden, daß es an ausdrücklichen Verfahrensvorschriften für die Verhandlung von Willkürverstößen fehlte und daß ihre Behandlung in der Gemeindeversammlung einen schriftlichen Niederschlag nicht gefunden hat.

Kehren wir also zur Normdurchsetzung in den Städten zurück. Nach der insbesondere von Wilhelm Ebel erforschten Theorie der Willkür und ihrer Durchsetzung ist mit der Ablegung des Eides der Einsatz der gesamten Persönlichkeit und ihres Vermögens verbunden. Der den Eid Leistende spricht sich damit für den Fall, daß er Willkür und Eid bricht, sein Urteil selbst. Es bedurfte folglich eines eigentlich gerichtlichen Verfahrens und eines dem Urteil entsprechenden gerichtlichen Erkenntnisses gar nicht. Bei vielen Fallgestaltungen mußte sich dies jedoch als reine Theorie erweisen. Nach den Maßstäben des überkommenen Rechts, deren Gerechtigkeitsgehalt ja nicht einfach außer Kraft gesetzt werden konnte, war auf einen wie auch immer gearteten Erkenntnisvorgang nämlich nur bei Offenkundigkeit/Notorietät des zu beurteilenden Sachverhaltes zu verzichten. Dies galt

$28 \mathrm{Vgl}$. Anm. 15 . 
auch für das Willkürverfahren des Rates ${ }^{29}$. Es mußten also in der Praxis zumindest die dem Vorwurf zugrunde liegenden Fakten festgestellt und es mußten hierfür auch Beweisregeln entwickelt werden. Späterhin leitete der Rat solche Untersuchungen ein, und er war es auch, der die Ergebnisse würdigte. Die Forschungen zum ausgebildeten Stadtrecht haben dies in hinreichender Weise belegt. Die Verhältnisse davor sind mir unbekannt. Man kann nur vermuten, daß die Gemeindeversammlung oder ihre Vorleute in vergleichbarer Weise tätig wurden.

Das Willkürverfahren des städtischen Rates nun zeigt noch schärfer und eindrucksvoller, als wir dies heute für den Bereich der materiellen Normen nachzuempfinden in der Lage sind, den mit der Willkür verbundenen Bruch der Rechtstradition auf. Das Ratsverfahren bringt ein gänzlich neues Gerichtsmodell herauf, nämlich die kollegiale Entscheidung ohne Gewaltenteilung und garantierte Gerichtsöffentlichkeit. Rechtsetzung, Rechtsfindung und Rechtszwang liegen in der Hand des Kollegialorgans Rat. Das Verfahren selbst ist im Prinzip rational, d. h. auf die Ermittlung der materiellen Wahrheit gerichtet. Die Entscheidungsfindung bietet dem Beschuldigten jedoch keinerlei Rechtsgarantien. Offenbar führten das älteste, bedeutendste Ratsmitglied oder der Bürgermeister nicht nur den Vorsitz, sondern auch die erste Stimme. Äußerungen in der Literatur deuten darauf hin, daß die Stimmabgabe der übrigen Ratsmitglieder durch diese erste Stellungnahme weitgehend festgelegt war. Das Verfahren des Rates wurde auch nicht ausreichend durch die Öffentlichkeit kontrolliert. Ging es um schweren Willkürbruch und Kriminalität, so war die Öffentlichkeit ausgeschlossen. Von hier aus führt eine strikte Linie zur Verwilderung des spätmittelalterlichen Strafrechts, insbesondere des Strafverfahrens. Das Ratsverfahren überlagerte das traditionelle Rechtsverfahren, bildete die Offizialmaxime aus, und mit ihm verband sich die gleichzeitig aus dem Kirchenrecht übernommene Folter zum städtischen Inquisitionsprozeß. In Zivilrechtsstreitigkeiten war die Öffentlichkeit des Ratsverfahrens beschränkt, d. h. die Parteien durften mitsamt einiger ihrer Freunde der Verhandlung beiwohnen. Die Entscheidungsfindung jedoch war offenbar überall - anders als in Verfahren nach Landrecht - geheim.

Dieses den Beschuldigten wohl unter Berufung auf die umfassende Wirkung des Bürgereides zugemutete Verfahren verband sich mit weiten Spielräumen hinsichtlich der materiellen Rechtslage. Der Bürgereid klärte die übernommenen Pflichten ja nicht in einer der modernen Tatbestandslehre entsprechenden Weise $\mathrm{ab}$, sondern postulierte allgemeine Anforderungen an das Wohlverhalten. Das Ratsverfahren kombinierte also, was die Verletzung des Bürgereides angeht, die weitgehend ungebundene Entscheidungsfindung eines mutmaßlich oligarchisch geprägten Organs mit einem prinzipiell ungebundenen Ermessen in materiellrechtlicher Hinsicht. Unter solchen Bedingungen war der gemeine Bürger dem Rat als Obrigkeit ausgeliefert. Wilhelm Ebel hat anhand etlicher grober Beispiele

29 Ebel, Willkür (wie Anm. 1), 56-63; ders., (wie Anm. 15), 352 ff.; Pirmin Spieß, Rüge und Einung dargestellt anhand süddeutscher Stadtrechtsquellen aus dem Mittelalter und der frühen Neuzeit (Speyer 1988); Weitzel, (wic Anm. 24), 135-147. 
auf die unter solchen Verhältnissen unvermeidbaren Auswüchse hingewiesen ${ }^{30}$. Er spricht von einer Pandorabüchse der Verwillkürung und des Statutarrechts, die der Rat nach Belieben öffnen und handhaben konnte. Aus dieser Büchse konnte freilich auch die Gnade entfliehen. Es sind folglich an dem gängigen Bild, das die Stadt als eine Art kleinen bürgerlichen Rechtsstaat zeichnet, doch erhebliche Abstriche vorzunehmen. Insgesamt gesehen ergibt sich der Eindruck, daß zumindest die deutsche Stadt ausgangs des Mittelalters auch ohne Rezeption die "Rechtstheorie", nach der sie angetreten war, weitgehend verlassen hatte.

\section{Statutarrecht und Kommune in Italien}

Charakter und Entwicklungen der in Norditalien aus der conjuratio hervorgehenden Normen stellen sich wesentlich anders dar. In Italien führten die Statuten keine neuartige Normstruktur herauf. Auch von einer in unterschiedlichen Formen organisierten und ausgeübten Gerichtsbarkeit ist mir nichts bekannt. Da das Recht in Fortführung antiker Vorstellungen den Rechtszwang in sich selbst trug, war das Urteil keine genossenschaftliche Aussage über die Rechtslage, die erst noch durch einen Herrn hätte geboten werden müssen ${ }^{31}$. Die Schwierigkeiten des Verhältnisses zwischen überkommenem Recht (in Italien Gesetz und Gewohnheitsrecht) und dem neuen Statutarrecht beschränkten sich folglich auf die rechtssystemimmanente Frage nach der Gesetzgebungskompetenz der Kommunen. Durch den Eintritt des Adels in die italienische Stadt, die Ausbildung der Signorie und die Entwicklung der Städte zu Flächenstaaten wurden die Statuten zur vorherrschenden Erscheinungsform des Rechts in Norditalien. Daher rührt es, daß das italienische Statutarrecht heute weitaus besser erforscht ist und in Theorien der Rechtsentwicklung einen weitaus gewichtigeren Platz einnimmt als das $\mathrm{Ge}$ wohnheitsrecht ${ }^{32}$.

Die potestas condendi statuta der Kommunen war also im Prinzip unproblematisch, hatte die Kommune erst einmal die politische Gewalt erlangt. Konkurrenzprobleme und Kompetenzstreitigkeiten gab es im Verhältnis zwischen Signorie und Stadtgemeinde einerseits, sowie zwischen der beherrschenden Stadt und der ihrem Gebiet eingegliederten (Land-)Stadt andererseits. Als die Interpreten des gemeinen Rechts sich der gesetzgebenden Gewalt der Kommunen zuwandten, war deren Statutargewalt längst ausgebildet und grundsätzlich anerkannt. Die von den Interpreten zum Thema potestas condendistatuta entwickelten Theorien waren allesamt nur nachträgliche theoretische Rechtfertigungen dessen, was man in der Wirklichkeit

30 Ebel, (wie Anm. 15), $360 \mathrm{ff}$.

31 Weitzel, (wie Anm. 7), $151 \mathrm{f}, 331 \mathrm{ff} ., 1120 \mathrm{ff}$.

32 Giorgio Chittolini, Statuten und städtische Autonomien. Einleitung, in: Statuten, (wie Anm. 15) 7-37, 7 f., 11. 
vorfand ${ }^{33}$. Es handelt sich also um Versuche, innerhalb des Corpus Juris Civilis die Grundlagen für eine als Rechtserscheinung unstreitige Autonomie zu finden. Die maßgeblichen Theorien wurden im 14. Jahrhundert von den Kommentatoren entwickelt. Auf die Einzelheiten kann hier nicht eingegangen werden. Im Prinzip ging es um die Interpretation von Digesten 1.1.9 (De iustitia et iure, lex Omnes populi): „Omnes populi, qui legibus et moribus reguntur, partim suo proprio, partim communi omnium hominum iure utuntur. Nam quod quisque populus ipse sibi ius constituit, id ipsius proprium civitatis est vocaturque ius civile, quasi ius proprium ipsius civitatis: quod vero naturalis ratio inter omnes homines constituit, id apud omnes peraeque custoditur vocaturque ius gentium, quasi quo iure omnes gentes utuntur." Bartolus bestimmte im Jahre 1343 die Statutarbefugnis der Stadtgemeinde ( $p o-$ pulus) durch die Jurisdiktion: „Omni populo iurisdictionem habenti, statuere permittitur quod ius civile vocatur." Damit war das Statutarrecht auf solche populi beschränkt, welche sich voll oder wenigstens teilweise im Besitz der iurisdictio befanden. Die nicht mit einer iurisdictio versehenen populi, wie die villae und die castra, die einer civitas oder einem dominus unterworfen waren, konnten in Angelegenheiten von allgemeiner Bedeutung ohne die Zustimmung ihres superior keine Beschlüsse fassen, da sie statutarische Befugnisse nur im Bereich der lokalen Verwaltung (ad administrationem rerum ipsius populi) innehatten. Bartolus erkannte auf der anderen Seite die Tatsache an, daß sich auch in den Städten, die im Besitz einer vollständigen iurisdictio waren, die Gültigkeit der Statuten als von der Zustimmung eines superior abhängig erweisen konnte. Neben den Gemeinden kam den iudices maiores (Senatoren, Präfekten) sowie den Grafen und Bischöfen die Macht zum Erlaß dauerhafter Gesetze zu. Hingegen konnten die städtischen Magistrate (Konsuln, Podestas) nur mit zeitweiliger Wirkung und meist auf den Bereich ihres Auftrags beschränkt statuieren. Mit dem Abstellen auf die iurisdictio hatte Bartolus die Statutarbefugnis der Gemeinden restriktiv interpretiert. Da der Erwerb und der Fortbestand der iurisdictio ihrerseits von weiteren Faktoren abhingen, erwies sich die Statutarbefugnis der Gemeinde unter Umständen selbst als zweifelhaft und gefährdet. Zudem bestand für die Gemeinden die Gefahr, daß auch die domini, die signori, im Wege der Interpretation in die Kategorie der indices maiores oder der Träger einer erblichen dignitas, denen eine auf Dauer gestellte Statutarbefugnis zukam, gerechnet werden könnten.

Dem Bartolus widersprach Ranieri Arsendi in seiner "Repetitio“. Er bestritt eine Verknüpfung zwischen iurisdictio und potestas condendi statuta und betonte die Vorrangstellung des consensus populi hinsichtlich der Ausbildung von Statuten. Die lex Omnes populi spreche nur undifferenziert von einem jeden Volk, das nach Schriftrecht und nach Gewohnheitsrecht lebe. Arsendi sagte zwar, daß der populus auch der Zustimmung seines superior bedürfe, um den eigenen Statuten

33 Claudia Storti Storchi, Betrachtungen zum Thema „Potestas condendi statua“, in: Statuten (wie Anm. 15), 251-270, 251 (auch zum Nachfolgenden); vgl. jetzt auch Wolfgang P. Müller, Signorolus de Homodeis and the Medieval Interpretation of Statutory Law, in: Rivista internazionale di diritto comune 6 (1995) 217-232. 
Geltung und Wirksamkeit zu verleihen. Doch diese Zustimmung konzipierte er als Mitwirkung in der Phase der Ausarbeitung des zu verabschiedenden Textes, während dessen Prüfung und Verabschiedung der Gemeinde allein zustehen sollte. Diese Theorie engte also die Befugnis der Gemeinden zur Statutargesetzgebung weit weniger ein als die des Bartolus.

Die beiden Theorien standen bis in das 16. Jahrhundert hinein miteinander in Widerstreit. In der Praxis setzte sich die engere Interpretation des Bartolus durch, die auch nördlich der Alpen rezipiert wurde. In der italienischen gemeinrechtlichen Wissenschaft des 16. Jahrhunderts erlebte die Theorie des Arsendi dann einen neuen Aufschwung, als es darum ging, verschärfte Angriffe auf und Eingriffe in das Statutarrecht der Gemeinde abzuwehren.

\section{Gebotsrecht und Kommune}

\section{Autonomes Gebotsrecht}

Zunächst darf daran erinnert werden, daß schon die hoch- und spätmittelalterliche Stadtgemeinde, wie vorgehend ausgeführt, in großem Umfange Gebotsrecht erlassen hat, das inhaltlich als Policey charakterisiert werden kann. Auch die übrige Statutargesetzgebung tendierte angesichts obrigkeitlicher Stadtregimente und zunehmender Verschriftlichung dazu, den Charakter einseitig gebotenen Rechts anzunehmen.

\section{Gemeines Recht und Kommunen}

Ob man zwischen dem gemeinen Recht und den Kommunen unter dem Gesichtspunkt des Gebotsrechtes Beziehungen zu entdecken sich bemüht, hängt zunächst einmal davon ab, ob man das auf der Grundlage des Corpus Juris Civilis sich entwickelnde gemeine Recht überhaupt als wirkliches Gebotsrecht anerkennt. Die Bedenken, die dagegen bestehen, wurden vorgehend bereits formuliert. Meines Erachtens ist dieser tradierte Normenkomplex, der im wesentlichen zivilrechtliche Regelungen anbietet, erst dem 18. Jahrhundert zum Gesetz in dem Sinne geworden, in dem wir heute von einem Gesetz sprechen. Teilt man diese Bedenken nicht, so stellt sich die Frage, inwieweit das gemeine Recht von seinen Inhalten her gesehen auf die Stellung der Kommune Einfluß gewinnen konnte. Über das von Bartolus entwickelte iurisdictio-Theorem hinausgehende Einflüsse sind insoweit jedoch nicht festzustellen. Die iurisdictio-Lehre bestimmte noch in der zweiten Hälfte des 17. Jahrhunderts die staatsrechtlichen Lehren über das städtische Gesetzgebungsrecht ${ }^{34}$. Diese Verknüpfung war also inzwischen in das ius publicum

3t Dietmar Willoweit, Kommunale Genossenschaften als Träger des Rechts in Mitteleuropa, in: Landgemeinde (wie Anm. 18), 403-423, 409 ff. 
übergegangen, während das gemeine Recht als solches für den Status der Gemeinden offenbar ohne Belang war.

\section{Ius Publicum, landesherrliche Policeyordnungen und Kommunen}

Das Rechtskonzept, das in der frühen Neuzeit schließlich die kommunale Autonomie dekonstituierte, ist das des entstehenden Territorialstaates, der flächendeckend Gebiete und Rechte sammelt und bündelt und schließlich zur Territorialgewalt zusammenfaßt. Nach den ersten Versuchen schon im 14. Jahrhundert nimmt dieser Prozeß in Deutschland im 15. Jahrhundert auf breiter Basis seinen Anfang. Zunächst geht es dabei nicht um das Hineinregieren in die Stadt, sondern in weiten Bereichen erst einmal darum, die Voraussetzungen dafür zu schaffen. Viele Städte müssen einer/ihrer Landesherrschaft überhaupt erst mal unterworfen werden. Dies geschieht anfangs, indem man ihre Gerichtsbarkeit in ein auf den Landesherrn hin ausgerichtetes System der Gerichtsverfassung einfügt $^{35}$. Die Unterwerfung der Ratsgerichtsbarkeit unter den zum landesherrlichen Hofgericht führenden Instanzenzug, das Abschneiden der Territorialgrenzen weiträumig übergreifenden Rechtszüge an auswärtige Oberhöfe und das Beschneiden der gerichtlichen Kompetenzen des Rates durch Aktivierung der verbliebenen stadtgerichtlichen Zuständigkeiten sind wesentliche frühe Merkmale der sich abzeichnenden Entwicklung. Indem die Städte in ihrer Jurisdiktion beschnitten und eindeutig als Landstadt eingestuft werden, verlieren sie auch einen Teil ihrer statutarischen Befugnisse. Zum Beispiel können sie hinfort nur noch im Rahmen überkommener Gewohnheiten statuieren. Die Übernahme und Durchsetzung der Instanzenzüge des gemeinen Rechts wie auch des von Bartolus entwickelten Jurisdiktionstheorems zeigen, in welchen geistesgeschichtlichen Zusammenhängen die Entwicklung zum Territorialstaat und dann zum Absolutismus wurzelt: Es handelt sich um Produkte der Rezeption des römisch-kanonischen Rechts, die eben nicht nur auf dem vielbeackerten Feld des Zivilrechts ihre Früchte trug, sondern auch das entstehende ius publicum nachhaltig prägte ${ }^{36}$. Während die autoritative Konzeption des gelehrten Rechts in zivilistischen Belangen durch die Brauchbarkeit der vom verblichenen Gesetzgeber aufgestellten Regeln entschärft war, schlug der Gesetzgebungstopos im Bereich des ius publicum voll durch. Hier - und nicht im Zivilrecht - ging es den angehenden Territorialherren darum, neue Strukturen durchzusetzen. Zugleich eröffnete sich in der nun aktivierten Verwaltung ein weites Tätigkeitsfeld, das nicht den überkommenen Strukturen der dinggenossenschaftlichen Rechtsgewohnheit verhaftet war.

35 Jürgen Weitzel, Wege zu einer hierarchisch strukturierten Gerichtsverfassung im 15. und 16. Jahrhundert, in: Akten des 26. Deutschen Rechtshistorikertages in Frankfurt am Main (Frankfurt a.M. 1987) 333-345.

36 Karl Kroeschell, Die Rezeption der gelehrten Rechte und ihre Bedeutung für die Bildung des Territorialstaates, in: Deutsche Verwaltungsgeschichte I (Stuttgart 1983) 279-288; Michael Stolleis, Geschichte des öffentlichen Rechts in Deutschland, I, 1600-1800 (München 1988) 58-63. 
Dieser Bereich der Ordnung und Verwaltung, auch der ordnenden und fortbildenden Einflußnahme auf das Recht ist das Exerzierfeld der späterhin ausschließlichen landesherrlichen Gesetzgebungshoheit. In den staatsrechtlichen und politikwissenschaftlichen Grundlagen der Policeygesetzgebung der frühen Neuzeit ist folglich die "Rechtstheorie“ $\mathrm{zu}$ sehen, die die Gemeindeautonomie bis zum 18. Jahrhundert hin endgültig dekonstituierte. Wie dies praktisch, politisch durchgesetzt wurde, haben jüngst wieder Beiträge in dem von Giorgio Chittolini und Dietmar Willoweit herausgegebenen Sammelband „Statuten, Städte und Territorien zwischen Mittelalter und Neuzeit in Italien und Deutschland" gezeigt. Um abschließend dem genius loci zu huldigen, sei insbesondere auf die Ausführungen von Hans Schlosser zur Niederwerfung Münchens und anderer bayerischer Städte durch die „landesherrliche Machtarroganz“ Kurfürst Maximilians I. in den Jahrzehnten um 1600 hingewiesen ${ }^{37}$.

37 Hans Schlosser, Statutarrecht und Landesherrschaft in Bayern, in: Statuten (wie Anm. 15), 177-194, $186 \mathrm{ff}$. 